\title{
Correction of edge-flame propagation speed in a counterflow, annular slot burner
}

\author{
Manh-Vu Tran, Min Suk Cha* \\ Clean Combustion Research Center, King Abdullah University of Science and Technology, Thuwal, 23955-6900, Saudi Arabia
}

\section{A R T I C L E I N F O}

\section{Article history:}

Received 16 August 2015

Revised 16 September 2015

Accepted 17 September 2015

Available online $\mathrm{xxx}$

\section{Keyword:}

Annular counterflow

Edge-flame

Displacement speed

Propagation speed

\begin{abstract}
A B S T R A C T
To characterize the propagation modes of flames, flame propagation speed must be accurately calculated. The impact of propagating edge-flames on the flow fields of unburned gases is limited experimentally. Thus, few studies have evaluated true propagation speeds by subtracting the flow velocities of unburned gases from flame displacement speeds. Here, we present a counterflow, annular slot burner that provides an ideal one-dimensional strain rate and lengthwise zero flow velocity that allowed us to study the fundamental behaviors of edge-flames. In addition, our burner has easy optical access for detailed laser diagnostics. Flame displacement speeds were measured using a high-speed camera and related flow fields of unburned gases were visualized by particle image velocimetry. These techniques allowed us to identify significant modifications to the flow fields of unburned gases caused by thermal expansion of the propagating edges, which enabled us to calculate true flame propagation speeds that took into account the flow velocities of unburned gases.
\end{abstract}

(C) 2015 The Combustion Institute. Published by Elsevier Inc. All rights reserved.
The propagation speeds of edge-flames need to be correctly measured with respect to the unburned gas mixture. Cha and Ronney [1] reported systematic propagation rates of non-premixed edge-flames in a two-dimensional slot burner, and propagating flames were assumed to have no effect on the flow fields of unburned gases. Thus, a displacement speed of flame edge was considered as a propagation speed of an edge-flame. In the present study, we design a new counterflow, annular slot burner that prevents both lengthwise convection and strain. In addition, its easy optical access facilitates various laser diagnostics that enabled us to achieve our goal of measuring the flow velocity ahead of propagating flame edge, allowing us to deduce edge-flame propagation speeds relative to unburned gas.

The counterflow, annular slot burner consisted of upper and lower parts, each with three co-axial annular slots (see Supplementary Material 1). Because of an axisymmetric configuration of the annular slots, we expected there to be no azimuthal velocity component along the slots in cold-flow conditions (i.e., zero strain rate on the annular slots in the azimuthal direction). In addition, once a flame stabilizes, there is no edge of the flame along the slot because they form a ringshaped flame within the burner. The main, central, annular slot had a length of $606 \mathrm{~mm}$. Nitrogen sheath flows occupied the two radially adjacent slots. Each slot was $2 \mathrm{~mm}$ wide, separated by $2 \mathrm{~mm}$ walls, and the gap $(d)$ between the upper and lower parts of the burner was

\footnotetext{
* Corresponding author.

E-mail address: min.cha@kaust.edu.sa, minsuk.cha7@gmail.com (M.S. Cha).
}

$9 \mathrm{~mm}$. The burner was divided into four sections $\left(90^{\circ}\right)$; each quarter may have different mixtures and/or flow velocities.

$\mathrm{CH}_{4} / \mathrm{N}_{2}$ and $\mathrm{O}_{2} / \mathrm{N}_{2}$ mixtures were injected $\left(U_{\text {jet }}\right)$ from the main slots of the lower and upper parts of the burner, respectively, producing a non-premixed flame. Global strain rates $(\sigma)$ of the unburned gas mixtures $\left(=2 U_{\text {jet }} / d\right)$ were fixed at $30 \mathrm{~s}^{-1}$ (i.e., $U_{\text {jet }}=13.5 \mathrm{~cm} / \mathrm{s}$ ) to observe moderately propagating edge-flames [1]. The velocities of the sheath flows were matched with $U_{\text {jet }}$. The level of nitrogen dilution $(Q)$ in a stoichiometric flame front $\left(\mathrm{CH}_{4} / \mathrm{O}_{2} / \mathrm{N}_{2}=1 / 2 / Q\right)$ was varied to control flame strength. We adopted a stoichiometric mixture fraction $Z_{\mathrm{st}}=0.5$ [1], such that the non-premixed flames could adequately be located on the stagnation plane. High-speed cameras measured the displacement speeds of edge-flames and a continuous wave Ar-ion laser and oil-based seed droplets were used for particle image velocimetry (PIV). Detailed experimental method can be found in Supplementary Material 1.

Figure 1 shows the measured flow fields of advancing (a) and retreating (b) edge-flames together with the superimposed images of the corresponding edge-flames (marked by black solid areas). Figure 1a shows the relatively stronger mixture having a smaller amount of nitrogen $(Q=6)$, where the flame pushes the flow ahead of the edge immediately, indicating very narrow thermal thickness between the flame edge and the area of detected velocity vectors (the seeded oil droplets evaporated at $\sim 230^{\circ} \mathrm{C}$ ). Even in the area directly behind the edge, the flow velocity created by the flame is still greater than the flow velocity supplied from the slots, resulting in diverging streamlines from the flame (see Supplementary Materials 2 and 3). 


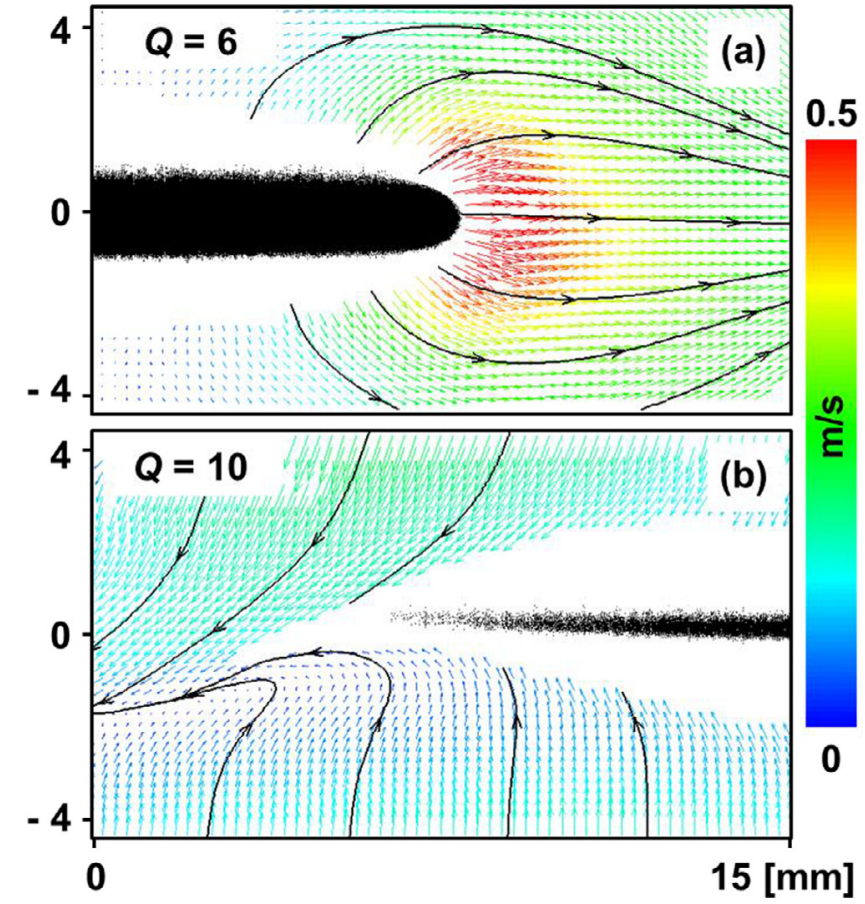

Fig. 1. PIV measurement of flow fields shows significant impact of both advancing (a) and retreating (b) edge-flames to the flow fields of the unburned gas. Superimposed solid black areas indicate propagating edge-flames moving from left to right. $Q$ refers to the level of nitrogen dilution as $\mathrm{CH}_{4} / \mathrm{O}_{2} / \mathrm{N}_{2}=1 / 2 / \mathrm{Q}$. Strain rate was fixed at $30 \mathrm{~s}^{-1}$ $\left(U_{\text {jet }}=13.5 \mathrm{~cm} / \mathrm{s}\right)$ for all test conditions.

Note the retreating edge in Fig. $1 \mathrm{~b}$ that moves to the right in the image while generating an unburned velocity component away from the flame edge. Thus, modifications to the unburned flow fields are not expected to be a function of the direction of propagation but instead due to thermal expansion. However, because there is a big gap between a retreating edge and a particle detected zone, which is crescent shaped far from the trailing edge, it was difficult to correct the displacement speed of the retreating edge with respect to the flow velocity of the unburned gases (see Supplementary Material 4).

As a result of analysis, the displacement speed $\left(U_{d}\right)$, the flow velocity of the unburned gases $\left(U_{u}\right)$, and the propagation speed with respect to the flow fields of the unburned gases $\left(U_{\text {edge }}=U_{d}-U_{u}\right)$ are shown in Fig. 2. Four runs of measurements were conducted for each $Q$ and the error bars represent standard deviations.

Displacement speed decreases with increasing nitrogen dilution level as expected in Ref. [1]. However, displacement speeds found here are smaller than those in Ref. [1], and the level of nitrogen dilution $(Q=10)$ to obtain a retreating edge at $\sigma=30 \mathrm{~s}^{-1}$ is much smaller (means stronger mixture) than $Q=11.75$ in Ref. [1]. These differences may partly be understood by variation in burner setups, including smaller slot width (2 $\mathrm{mm}$ in the present study), compared to that (5 mm) in Ref. [1].

The flow velocity of the unburned gas was defined as a horizontal maximum flow velocity. As shown in Fig. 2, the flow velocity of the unburned gas also decreases with increasing $Q$. This result was expected because weaker mixtures having larger $Q$ result in lower flame temperatures and thus, a reduced effect of gas expansion compared to that with higher flame temperatures. Once again, we could not correlate the retreating edge with $U_{u}$ as discussed previously,

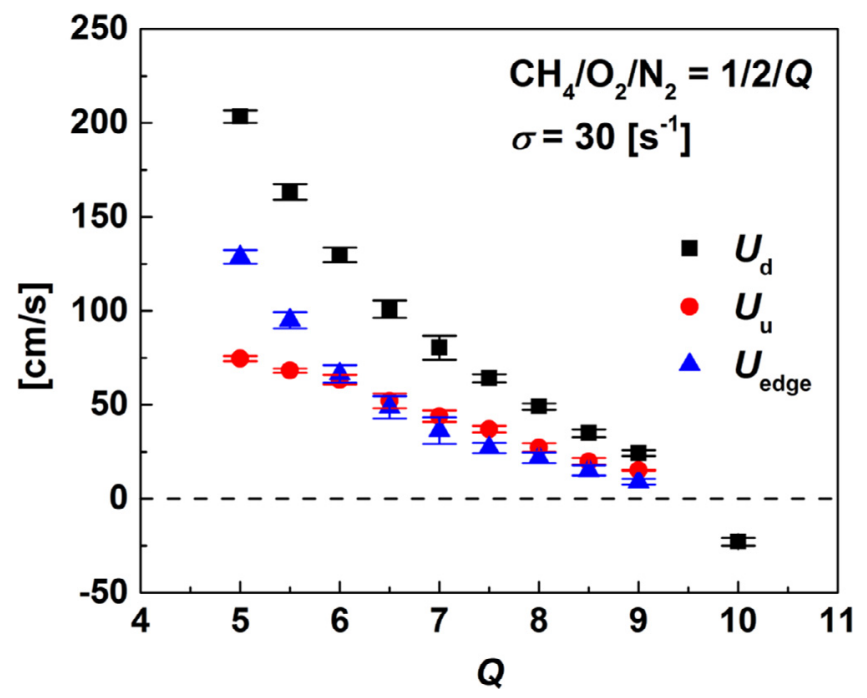

Fig. 2. Measured displacement speed of edge-flames $\left(U_{d}\right)$ and unburned flow velocity $\left(U_{u}\right)$ for various $\mathrm{CH}_{4} / \mathrm{O}_{2} / \mathrm{N}_{2}$ mixtures. Edge-flame propagation speeds $\left(U_{\text {edge }}=U_{d}-U_{\mathrm{u}}\right.$ ) corrected with respect to the flow velocity of the unburned gases are presented. $Q$ refers to the level of nitrogen dilution as $\mathrm{CH}_{4} / \mathrm{O}_{2} / \mathrm{N}_{2}=1 / 2 / \mathrm{Q}$. Strain rate was fixed at $30 \mathrm{~s}^{-1}\left(U_{\text {jet }}=13.5 \mathrm{~cm} / \mathrm{s}\right)$ for all test conditions.

thus there is no corrected propagation speed for the retreating edge $(Q=10)$.

Figure 2 illustrates the importance of the correction to quantitative analysis, and that higher precision is necessary to work with a finite-sized burner gap, which may limit the buffer space for thermal expansion in both experimental and numerical studies. In addition, it should be noted for a simulation that the inlet velocity boundary conditions (BCs) at the slot exit have to be selected carefully to reflect a drastic modification to the flow fields caused by the flame. A numerical approach for the BCs should be similar to that done for small laminar jet diffusion flames [2], where the importance of inlet boundary location has been reported.

In summary, we present a counterflow, annular slot burner capable of overcoming the typical limitations of two-dimensional counterflow slot burners. In this study, the displacement speeds of $\mathrm{CH}_{4} / \mathrm{N}_{2}-\mathrm{O}_{2} / \mathrm{N}_{2}$ non-premixed edge-flames were measured by collecting positions of flame edge as a function of time and the flow fields of the unburned gases were measured using PIV. We found noteworthy modifications to the flow fields of the unburned gases caused by a propagating flame edge (both advancing and retreating), and we report on the adjustment of propagation speeds of edgeflames with respect to unburned gases. We also note that it should be cautious to determine a propagation speed in lab scale experiments, because the modification of unburned flow field is apparatusdependent.

\section{Acknowledgments}

Research reported in this publication was supported by Competitive Research Funding from King Abdullah University of Science and Technology (KAUST).

\section{References}

[1] M.S. Cha, P.D. Ronney, Combust. Flame 146 (2006) 312-328.

[2] Y. Xiong, M.S. Cha, S.H. Chung, Proc. Combust. Inst. 35 (2015) 873-880. 